\title{
Los agentes de la investigación educativa en Chihuahua
}

\author{
Sandra Vega Villarreal \\ Universidad Pedagógica Nacional 081 \\ svegavillarreal@gmail.com
}

\begin{abstract}
Resumen
El presente documento forma parte de un diagnóstico estatal de la investigación educativa en Chihuahua. Se trata de un estudio estadístico descriptivo sobre los agentes que están produciendo en este campo. El análisis centra su atención en la cantidad de productos que reportan, lo cual, permite identificar los niveles de consolidación en el quehacer investigativo. Otros aspectos que se abordan son la antigüedad en el campo y de las áreas de conocimiento donde incursionan, así como las formas en que producen y los medios que utilizan para difundir su producción. El estudio muestra que de un total de 646 agentes registrados, únicamente 38 están activos en el campo trabajando tanto en forma individual como en grupos. La mayoría de estos agentes dispersa su producción en dos o tres áreas de conocimiento y difunden su producción generalmente a través de ponencias y escasamente a través de libros. Una gran parte de estos agentes produce sólo en su institución de pertenencia. Las instituciones que reportan más agentes de la investigación educativa son Universidad Autónoma de Chihuahua y el Centro de Investigación y Docencia.
\end{abstract}

Palabras clave

Diagnóstico estatal, investigación educativa, agentes.

\section{Objeto de estudio}

El presente estudio partió de la pregunta esencial ¿Cuáles son las condiciones que guarda la investigación educativa en Chihuahua a partir de los agentes que la producen? El objetivo principal fue realizar un acercamiento que evidenciara los agentes activos en el campo y las instituciones en donde se encuentran produciendo. A partir de la producción de los agentes, su antigüedad en el campo y de las áreas de conocimiento donde incursionan, se identifican los niveles de consolidación en el quehacer investigativo. Otros aspectos estudiados en la categoría de agentes son las formas en que producen y los medios que utilizan para difundir su producción.

El criterio para delimitar la categoría de agente de la investigación educativa fue incluyente, se tomó como única condición tener algún producto de investigación educativa en la entidad. Para acceder a estos datos se pusieron al alcance de los investigadores diversos medios para que reportaran su producción, mismos que se hicieron explícitos a través de una convocatoria pública a participar en el presente diagnóstico.

La metodología utilizada fue un acercamiento cuantitativo, descriptivo, que a partir de datos estadísticos permitió hacer interpretaciones fundamentales sobre los agentes que están produciendo investigación en Chihuahua.

\section{Resultados}

Agentes y su producción

Reconociendo que la consolidación de un agente en el campo de la investigación depende de una multiplicidad de factores, 
entre los que destacan el capital simbólico, institucional y social entre otros, se realiza una caracterización de los mismos a partir de su producción, por ser los datos directos arrojados por las fuentes de consulta. La producción generada permite ubicar a los agentes en cuatro categorías: potenciales, en desarrollo, en consolidación y consolidados. Los agentes potenciales de la investigación se definen como aquellos que registraron uno o dos productos (que en su mayoría son una tesis de maestría y una ponencia). Estos agentes se consideran potenciales pues aunque han vivido una experiencia de investigación aún no han incursionado en el campo.

Los agentes en desarrollo son aquellos que ya han incursionado de manera incipiente en el campo de la investigación, así que registran entre tres y seis productos, que en una generalidad se traducen en tesis de posgrado, ponencias y algunos artículos de revista.

Como agentes en consolidación se denomina a aquellos que registran entre siete y nueve productos de investigación. Se les considera en consolidación por su permanencia y debate en el campo, pues algunos de ellos tienen pocos años de incursionar en el ámbito de la investigación educativa cuentan con una diversidad de productos a través de los cuales establecen el debate con sus pares.

Finalmente, se clasifica como agentes consolidados a aquellos que registran diez o más productos de investigación. Generalmente son investigadores que tienen mayor antigüedad en el campo y han difundido su producción por una gran diversidad de medios. Algunos de ellos cuentan con reconocimiento social e institucional por su quehacer, pues perteneces al Sistema Nacional de Investigadores y al Consejo Mexicano de Investigación Educativa.

La información arrojada registra un total de 646 agentes de la investigación educativa en el estado de Chihuahua, mismos que se de acuerdo a la clasificación anterior se distribuyen de la siguiente manera:

Tabla 1. Agentes de la investigación y su producción.

\begin{tabular}{|c|c|c|}
\hline $\begin{array}{c}\text { AGENTES DE LA } \\
\text { INVESTIGACIÓN }\end{array}$ & PRODUCTOS REPORTADOS & TOTAL \\
\hline Potenciales & $1-2$ & 608 \\
\hline En desarrollo & $3-6$ & 24 \\
\hline En consolidación & $7-9$ & 9 \\
\hline Consolidados & 10 ó más & 5 \\
\hline TOTAL & & $\mathbf{6 4 6}$ \\
\hline
\end{tabular}

La tabla anterior muestra que el $94 \%$ de los agentes registrados a través de las diversas fuentes de información son un potencial que pudiera aprovecharse para el impulso de la investigación educativa en el estado, pues aunque han incursionado en alguna experiencia de investigación no se dedican a este quehacer como actividad fundamental. Ante la magnitud de esta población, se podría afirmar que en la entidad solamente el 6\% realiza investigación dentro de sus funciones académicas. Por tanto, en las siguientes 
categorías se trabajará sólo con los 38 agentes que realizan labores de investigación propiamente dichas.

\section{Agentes y dispersión de la producción}

En este apartado se analiza la incursión de los agentes en las diversas áreas de conocimiento. Como se señaló anteriormente, sólo se trabajará con los 38 agentes que tienen más de tres productos, puesto que aquellos que presentan uno o dos productos pudieran caracterizarse de manera superficial como agentes con continuidad en un área temática definida, lo cual es un dato engañoso en su apariencia, dado que la supuesta continuidad se debe a la precariedad de la producción, más que al desarrollo investigativo. Para ver la dispersión de la producción de los agentes, consúltese la siguiente tabla:

Tabla 2. Agentes de la investigación y su dispersión por las áreas temáticas

\begin{tabular}{|c|c|}
\hline No. DE ÁREAS DE INCURSIÓN & No. DE AGENTES \\
\hline 1 & 6 \\
\hline 2 & 11 \\
\hline 3 & 13 \\
\hline 4 & 3 \\
\hline 5 ó Más & $\mathbf{3 8}$ \\
\hline TOTAL & \\
\hline
\end{tabular}

El análisis sobre la dispersión de los agentes de la investigación educativa a partir del número de diferentes áreas de conocimiento en que incursiona, muestra que son muy escasos quienes mantienen consistencia en su campo de estudio; es decir que producen en un área. Igualmente, atípicos en la distribución son los agentes que producen en cuatro o más áreas de conocimiento, ya que la mayoría de la población se encuentra produciendo en dos o tres áreas diferentes. Este resultado brinda elementos para un análisis grueso sobre la poca consistencia del debate al interior del campo, puesto que la dispersión en la producción es un indicio de la falta de consolidación de los agentes en un área determinada, pues el transitar por diversos campos temáticos impide la construcción de objetos de estudio significativos o su desarrollo a profundidad.

Agentes y la difusión de su producción Los productos de investigación que presentan los agentes permiten conocer su actividad al interior del campo. El tipo de producción da cuenta si están participando activamente en el debate nacional o si la difusión de su producción se restringe al ámbito institucional.

El análisis estadístico del tipo de productos que reportan los agentes no coincide con el número de éstos, puesto que todos ellos registran más de tres productos, que en su mayoría son distintos medios de difundir su producción. Por tanto, solamente se esboza el número de investigadores que reportan determinado producto. 
Tabla 3. Los agentes de la investigación y la difusión de sus productos

\begin{tabular}{|l|c|}
\hline \multicolumn{1}{|c|}{ TIPO DE PRODUCTO } & $\begin{array}{c}\text { INVESTIGADORES QUE LOS } \\
\text { PRESENTAN }\end{array}$ \\
\hline Ponencias & 38 \\
\hline Capítulos de libro & 6 \\
\hline Cuadernos de trabajo & 1 \\
\hline Artículos & 14 \\
\hline Informes de investigación & 27 \\
\hline Libros & 10 \\
\hline
\end{tabular}

La distribución anterior muestra que todos los agentes debaten en el campo a través de ponencias en diversos espacios según su campo de producción, por lo que esta es la forma de difusión más utilizada.

Otro producto que reportan la mayoría de los investigadores son los informes de investigación dentro de los cuales se agrupan las investigaciones institucionales y tesis, dado que, finalmente tienen que concretarse en un informe.

Un medio importante para difundir la producción investigativa es la publicación de libros, pero sólo una cuarta parte de los agentes la utiliza. Habría que indagar las condiciones personales del investigador y las internas del campo de investigación que no facilitan el acceso de los agentes a la publicación de libros. Dentro del rubro de las publicaciones, son aún más escasos los autores de libros colectivos, pues escasamente se reportan capítulos de libro.

Aunque la publicación de artículos pareciera una forma común de difundir la producción, los resultados del presente diagnóstico muestran que una tercera parte de los agentes de la investigación reporta este tipo de productos, así es que no es muy común la difusión en revistas de la investigación generada. De manera incidental se registra el cuaderno de trabajo como forma de difusión, lo cual no implica que los agentes no realicen este tipo de productos, pero no lo reportan como producto de investigación.

Los agentes y las formas de producción en el campo

Tratando de identificar a los investigadores que producen en solitario, así como a los grupos de investigadores, $y$ tribus institucionales que están presentes en el campo, se realizó un análisis de la forma en que producen los agentes y los resultados fueron los siguientes: 
Tabla 4. Los agentes de la investigación y las formas de producción

\begin{tabular}{|l|c|}
\hline FORMAS DE PRODUCCIÓN & AGENTES \\
\hline Individual & 8 \\
\hline Grupal & 3 \\
\hline Individual / grupal & 27 \\
\hline TOTAL & $\mathbf{3 8}$ \\
\hline
\end{tabular}

forma de producir no es exclusiva de ninguna

El análisis estadístico de la tabla muestra que la gran mayoría de los investigadores produce tanto de forma individual como en grupos. Al tratar de identificar los grupos de investigación se encuentra que la mayoría se agrupa con colegas de su institución para producir colectivamente. Son muy escasos los investigadores que producen en solitario. Igualmente, reducido es el grupo de agentes que reporta productos grupales, principalmente ponencias que presentan en conjunto con sus estudiantes de posgrado.

Entre quienes producen en solitario se encuentran agentes en desarrollo, en consolidación y consolidados, así que esta categoría. En cambio, en la forma de producción grupal, se encuentra que en su mayoría son agentes en desarrollo que producen en conjunto con colegas de su institución.

\section{Agentes y su permanencia en el campo}

Para determinar la permanencia de los agentes en el campo de la investigación educativa, se partió desde el periodo en que registra su producto más antiguo hasta el 2011. La siguiente tabla muestra la distribución de los agentes según el tiempo de permanencia en el campo.

Tabla 5. Agentes y el promedio de su permanencia en el campo

\begin{tabular}{|l|r|}
\hline CATEGORÍA DE LOS & PROMEDIO PERMANENCIA EN AÑOS \\
\hline En desarrollo & 8.96 \\
\hline En consolidación & 12.22 \\
\hline Consolidados & 16.60 \\
\hline Promedio General & $\mathbf{1 2 . 5 9}$ \\
\hline
\end{tabular}

El promedio general de la permanencia de los agentes en el campo es de 12 años y medio. Al hacer un análisis más fino, se puede apreciar que la antigüedad se encuentra en relación directa entre la categoría en que aparece. Los agentes en desarrollo su promedio de antigüedad en el campo es de casi nueve años, los agentes en consolidación se acercan a los doce años produciendo en el campo y los agentes consolidados han participado por más de dieciséis años.

Tratando de establecer una relación entre la categoría de los agentes y su permanencia en el campo se realizó un desglose más fino de los datos a partir de una serie de intervalos que permitieran establecer 
la periodización de actividad científica de los agentes en un rango de 27 años, calculando los agentes se evidencia en la siguiente tabla. intervalos en lustros. La distribución de los

Tabla 6. Categoría del agente y su antigüedad en el campo.

\begin{tabular}{|c|c|c|c|c|c|}
\hline AGENTE & 1 A 5 & 6 A 10 & $\begin{array}{l}11 \mathrm{~A} \\
15\end{array}$ & $\begin{array}{l}16 \mathrm{O} \\
\text { MÁS }\end{array}$ & TOTAL \\
\hline En desarrollo & 6 & 11 & 3 & 4 & 24 \\
\hline En consolidación & 0 & 3 & 3 & 3 & 9 \\
\hline Consolidado & 0 & 0 & 2 & 3 & 5 \\
\hline TOTAL & 6 & 14 & 8 & 11 & 38 \\
\hline
\end{tabular}

El análisis a partir de las medias muestra que existe una relación directamente proporcional entre las categorías de agentes y el promedio de su antigüedad en el campo. A mayor nivel de consolidación del agente, mayor número de años de permanencia en el campo. Sin embargo, un análisis estadístico en términos absolutos permite ver que la relación lineal que se establece a partir de las medias anteriores no es como aparenta, puesto algunos agentes cuya incursión en el campo es reciente, tienen una cantidad de productos que los ubica como agentes en consolidación y consolidados. Y viceversa, existen agentes que tienen una antigüedad considerable y su producción no es muy amplia.

Agentes de la investigación e instituciones de pertenencia

Las instituciones inciden de forma directa en la práctica de los agentes de la investigación educativa, a partir de las condiciones que generan para el desarrollo de su quehacer. "De allí que entre más orientada está la institución hacia la IE, más reconocimiento tendrá en el campo; y entre más agentes del campo agrupe, mayor será el espacio de socialización que proporcione a sus agentes. Es decir, favorecerá el crecimiento de un capital social en ellos y determinará el desarrollo de un estilo de pensar y sentir la IE, que caracterizará el trabajo profesional de los agentes" (Colina \& Osorio, 2003, p. 107).

La pertenencia a determinado número de instituciones permite identificar la trayectoria de los agentes, así como tener un acercamiento a las posiciones adquiridas por los agentes al interior del campo, dado que puede ser considerado un indicador de su exclusividad en la actividad de investigación o el desempeño de otras funciones como la docencia y la difusión. Para el análisis de este aspecto, se distribuyeron los agentes según su categoría y la cantidad de instituciones en las que producen $o$ han ha producido investigación. 
Tabla 7. Agentes de la investigación e instituciones en las que produce

\begin{tabular}{|l|l|l|l|l|}
\hline \multirow{2}{*}{ AGENTES } & \multicolumn{5}{c|}{ CANTIDAD INSTITUCIONES EN LAS QUE } \\
& \multicolumn{5}{|c|}{ PRODUCE } \\
\cline { 2 - 5 } & $1-2$ & $3-4$ & $5-6$ & TOTAL \\
\hline En desarrollo & 22 & 2 & 0 & 24 \\
\hline En consolidación & 4 & 4 & 1 & 9 \\
\hline Consolidados & 2 & 1 & 2 & 5 \\
\hline TOTALES & $\mathbf{2 8}$ & $\mathbf{7}$ & $\mathbf{3}$ & $\mathbf{3 8}$ \\
\hline
\end{tabular}

La tabla anterior muestra que la dispersión de los agentes de la investigación educativa en las diferentes instituciones de educación superior en Chihuahua no es muy amplia, pues el $74 \%$ de los agentes participan en una o dos instituciones, el 18\% reparte su producción entre 3 y 4 instituciones y apenas el $8 \%$ tiene sus actividades completamente dispersas en 5 o 6 instituciones. También se observa que los agentes en desarrollo en su generalidad, producen investigación en una sola institución, mientras que los consolidados y en consolidación transitan por diversas instituciones.
Tratando de proporcionar un panorama general de los agentes y las instituciones donde están insertos, se organizan los datos obtenidos a través de las cuatro fuentes de información ya mencionadas al inicio de este documento. La población estudiada son los 38 agentes que están participando activamente en el campo de la investigación educativa, distribuida según su nivel de producción en las instituciones donde se encontraban ubicados hasta el 2011. Véase la siguiente tabla:

Tabla 8. Agentes de la investigación e instituciones de adscripción

\begin{tabular}{|c|c|c|c|c|}
\hline \multirow[t]{4}{*}{ INSTITUCIÓN } & \multicolumn{4}{|c|}{ AGENTES } \\
\hline & EN & & CONSOLIDADO & \\
\hline & DESARROLLO & EN CONSOLIDACIÓN & $\mathrm{S}$ & \\
\hline & & & & TOTAL \\
\hline CCHEP & 5 & 0 & 1 & 6 \\
\hline CID & 5 & 3 & 1 & 9 \\
\hline IByCENECH & 1 & 0 & 0 & 1 \\
\hline ITESM & 1 & 1 & 0 & 2 \\
\hline UACH & 4 & 3 & 2 & 9 \\
\hline UACJ & 1 & 0 & 1 & 2 \\
\hline UPN & 3 & 2 & 0 & 5 \\
\hline SECYD & 2 & 0 & 0 & 2 \\
\hline NORMAL & & & & \\
\hline SAUCILLO & 1 & 0 & 0 & 1 \\
\hline INDEPENDIENTE & 1 & 0 & 0 & 1 \\
\hline TOTAL & 24 & 9 & 5 & 38 \\
\hline
\end{tabular}


La tabla anterior muestra que la UACH y al CID son las instituciones que cuentan con un mayor número de investigadores educativos debatiendo en el campo, según la producción reportada para este diagnóstico. Con un número importante de investigadores se encuentran la UPN y el CCHEP. En el caso específico de UPN se encuentra que algunos agentes adscritos en otras instituciones también forman parte ella, la razón de no considerarlos dentro de su plantilla es que dichos agentes no han registrado sus productos como generación institucional o no la reconocen como su institución de procedencia.

El resto de las instituciones enlistadas cuenta con un número muy precario de agentes de la investigación, oscilan entre uno y dos, así que la diferencia con las cuatro instituciones que agrupan al grueso de ellos es bastante considerable.

Un aspecto importante que se puede rescatar dentro del análisis del capital institucional de los agentes estudiados, es la pertenencia a organismos que otorgan un reconocimiento social en las tareas de investigación, tales como el Sistema Nacional de Investigadores (SNI) y el Consejo Mexicano de la Investigación Educativa (COMIE). No obstante, este rubro no se desglosa a profundidad en este apartado por la escases de agentes que pertenecen a estas instancias académicas, pues sólo dos de los 38 agentes pertenecen a ambos organismos, y otros dos son miembros sólo del COMIE, de tal manera que el reconocimiento social de los agentes que realizan investigación educativa en el estado de Chihuahua es muy precario en el contexto nacional.

\section{Referencias}

Aguirre Lares María Silvia, Argelia Antonia Ávila Reyes y Romelia Hinojosa Luján (2007), Aportes para el debate sobre el estado del conocimiento en el campo género y educación, revista Acoyahu, número 39 , pp. 26 - 35.

Colina, A., \& Osorio, R. (2003). Los agentes de la investigación educativa en México. In E. Weiss (Ed.), El campo de la investigación educativa 1993-2001. México: Consejo Mexicano de Investigación Educativa.

Hinojosa, R. (2010). Miradas a la educación desde el género (Vol. 7). Chihuahua, México: Secretaría de Educación y Cultura.

Latapí Sarre, Pablo (1994), La investigación educativa en México, México, Fondo de Cultura Económica, $243 \mathrm{pp}$.

Martínez, R. (2010). La Investigación Educativa. Su epistemología y sus métodos Vega, S. (2010). El campo lenguas en la investigación educativa (Vol. 4). Chihuahua, México: Secretaría de Educación y Cultura. 